THP-11(T2)

\title{
Visualizing Inflammatory Activity in Isolated Perfused Rat Hearts
}

\author{
Long-Sheng Lu \\ Graduate Institute of Pharmacology, National Taiwan University College of \\ Medicine \\ 1 Section 1 Jen-Ai Road, Taipei Taiwan \\ telefax: 886-2-23971403, email: lslu@pie.com.tw \\ Yen-Bin Liu and Chau-Chung Wu \\ Department of Internal Medicine, National Taiwan University Hospital and \\ National \\ Taiwan University \\ 7 Chung-Shan South Road, Taipei, Taiwan \\ Chia-Wei Sun \\ Biomedical Engineering Center, Industrial Technology and Research Institute \\ Building 53, 195 Section 4, Chung Hsing Road, Chutung, Hsinchu, Taiwan
}

\begin{abstract}
Inflammation is a defense mechanism against various noxious stimuli. The recruitment of host leukocytes to sites of injury results in increased regional microvascular leakage and reactive oxygen species (ROS) generation. Excessive inflammatory activity not only eliminates offending stimuli but also result in tissue damage, as evidenced in reperfusion injury of the heart. To investigate spatial-temporal evolution of acute inflammation after myocardial reperfusion injury, we monitored microvascular leakage and reactive oxygen species generation with optical mapping technique. Reperfusion injury was performed on isolated blood-perfused rat heart, and it was labeled with dihydroethidium and large molecular weight tetramethylrhodamine conjugated dextran. Tissue was illuminated with a 532 $\mathrm{nm}$ laser, and epifluorescence at 580 and $650 \mathrm{~nm}$ was collected through 2 separate band pass filters. Our results indicate that 1. Optical mapping of myocardial inflammation is feasible; and 2. Reperfusion injury elicits substantial microvascular leakage and ROS production.
\end{abstract}

